\title{
Comment appréhender une tuerie de masse pour les équipes Smur primo-intervenantes?
}

\author{
How Should Prehospital Medical Unit Cope with Mass Shooting?
}

\author{
Service médical du RAID - Antenne médicale spécialisée de Satory (GIGN) - F. Lapostolle - T. Loeb · \\ E. Lecarpentier - B. Vivien - P. Pasquier - M. Raux \\ Reçu le 1 juin 2018; accepté le 13 août 2018 \\ (C) SFMU et Lavoisier SAS 2018
}

Résumé Le risque terroriste présent sur l'ensemble de notre territoire depuis plusieurs années conduit les équipes de Samu-Smur à intervenir sur les lieux d'une tuerie de masse aux côtés de forces de sécurité, voire en tant que primointervenants. De telles circonstances exposent les équipes à

Service médical du RAID

(Recherche, Assistance, Intervention, Dissuasion),

Force d'intervention de la Police nationale,

Domaine du Bel Air, route de Gisy, F-91570 Bièvres, France

Antenne médicale spécialisée de Satory (GIGN)

Première antenne médicale spécialisée,

Groupe d'intervention de la Gendarmerie nationale,

34, rue de la Martinière, F-78000 Versailles, France

F. Lapostolle

Samu 93, EA 3509, UFR Enseignement-Qualité,

hôpital Avicenne, université Paris-XIII, Sorbonne-Paris-Cité,

125, rue de Stalingrad, F-93009 Bobigny, France

T. Loeb

Samu 92, hôpital Raymond-Poincaré, AP-HP,

F-92380 Garches, France

E. Lecarpentier

Samu 94, hôpital Henri-Mondor, AP-HP, F-94010 Créteil, France

B. Vivien

Samu de Paris, hôpital Necker-Enfants-malades,

AP-HP, université Paris-Descartes, F-75015 Paris, France

P. Pasquier

Fédération anesthésie, réanimation, brûlés et blocs opératoires,

hôpital d'instruction des armées (HIA) Percy,

plateforme hospitalière militaire Île-de-France,

F-92140 Clamart, France

M. Raux $(\bowtie)$

Sorbonne université UMRS Inserm 1158,

département d'anesthésie-réanimation,

groupe hospitalier Pitié-Salpêtrière-Charles-Foix, AP-HP,

F-75013 Paris, France

e-mail : mathieu.raux@aphp.fr un environnement de travail inhabituel et hostile. Cette mise au point, proposée en complément de la recommandation formalisée d'experts sur la prise en charge de tuerie de masse, répond aux interrogations qui naissent sur le terrain en pareilles circonstances. Elle facilite une juste appréciation des événements, aide à réguler ses émotions, dans le but d'augmenter la capacité des soignants à agir, tout en réduisant la phase de sidération initiale, elle-même responsable d'une inertie à même d'impacter le pronostic des blessés en choc hémorragique. Des procédures anticipées, sous forme de check-lists, guident les actions à mener de manière sereine. Les équipes médicales préhospitalières doivent apporter sur le terrain non seulement une compétence médicale, mais également une compétence tactique et décisionnelle pour accélérer les flux d'évacuation. Le premier médecin engagé doit aider à la décision aux côtés du commandant des opérations de secours, du commandant des opérations de police et de gendarmerie et des médecins intégrés aux forces de sécurité intérieure en attendant le directeur des secours médicaux. Il doit donc également faire preuve d'une compétence de chef d'équipe.

Mots clés Médecine préhospitalière · Tuerie de masse · Médecine tactique
Abstract France faces terrorism for many years. Recent ter- ror attacks, as Paris on November 13, 2015, required prehos- pital medical teams (Smur) as well as medical dispatching (Samu), to work together with police and SWAT teams. Smur teams had to cope with unusual and hostile environ- ment. This unusual environment generated fear in prehospi- tal medical teams. This article aims at providing answers to justified questions that arise in such circumstances. It comes along with recent French guidelines that specify the prehos- pital organization when treating terrorist attack. It helps per- forming an adequate assessment of the scene. Moreover, it is committed to provide tricks to enhance personal emotional 
control for a better efficiency. Indeed, initial emotional sideration may slow down patients' rescue. Check lists help prehospital physician to perform all the tasks. The prehospital physicians not only provide medical assistance, but also provide tactical and operational competences to enhance casualties' evacuation toward safety nests. The very first physician must be competent to act as a pre-chief medical officer and collaborate with chief officer and police officer until chief medical officer arrives.

Keywords Mass causualty incident - Tactical medecine · Prehospital medecine

\section{Introduction}

Les acteurs du soin préhospitalier ainsi que ceux du secours ont conscience de la difficulté d'intervenir auprès d'un blessé lors d'une attaque terroriste [1,2]. Quel que soit sa fonction ou son grade, la réalité du terrain impose à chaque soignant une dualité : prendre en charge les victimes et se protéger d'une menace non maîtrisée. Toutefois, les chasubles et uniformes n'étant ni des vêtements de camouflage ni à l'épreuve des projectiles, la prégnance de la menace impose de se soumettre à des règles de sécurité avant de porter secours. En effet, le secours et le soin doivent être précédés d'une maîtrise de la menace $[3,4]$, phase chronophage, mais qu'il faut impérativement respecter, même si le temps qui s'écoule avant l'hémostase chirurgicale joue toujours en défaveur du patient.

Toutes les doctrines et tous les référentiels qui ont vu le jour depuis 2015 vont dans le même sens : permettre des actions de secours et de soins combinées à la neutralisation de la menace ${ }^{1}$.

L'objectif de cette mise au point est de répondre aux questions les plus fréquemment posées par les médecins préhospitaliers lors de la phase initiale de leur intervention. Sans prétendre leur garantir une absolue sérénité, ce texte ambitionne de donner quelques « clés » afin de mieux appréhender ces situations et de garantir une efficacité opérationnelle.

Cette mise au point a été rédigée par les médecins des deux forces d'intervention à compétence nationale (Recherche, Assistance, Intervention, Dissuasion [RAID] et Groupe d'intervention de la Gendarmerie nationale [GIGN]) et des

\footnotetext{
${ }^{1}$ Ministère de l'Intérieur. Instruction générale du 19 avril 2016 (NORINTC 1610640J) relative à l'intervention de la Police nationale dans un contexte de tuerie de masse (diffusion restreinte). Ministère de la Défense. Instruction du 6 juin $2017\left(\mathrm{n}^{\circ} 78000 / \mathrm{GEND} / \mathrm{DOE} / \mathrm{SDDOPP} /\right.$ $\mathrm{BDSN} / \mathrm{DR}$ ) relative à la réponse opérationnelle de la gendarmerie en cas d'attaque terroriste (diffusion restreinte). Ministère de l'Intérieur. Note de doctrine du 20 mars 2017 (DGSCGC/CAB/DSP/SDDRH/BDFE) relative à la réponse opérationnelle des services d'incendie et de secours en cas de tuerie de masse (diffusion restreinte).
}

experts de la médecine d'urgence préhospitalière. Elle partage l'expérience militaire du sauvetage au combat et l'expérience civile des mass casualty incident. Elle ne se substitue pas aux recommandations formalisées d'experts (« Prise en charge de victimes de tueries de masse ») [5], mais les complète en apportant des éléments clés de comportement lors de la phase tout initiale de l'intervention et en impactant les décisions prises en régulation médicale dès réception de l'alerte.

\section{Que dois-je faire pour me préparer avant mon arrivée sur place ?}

\section{Anticiper la phase initiale d'incertitude majeure}

Au premier stade de la crise, l'analyse de la situation et le dimensionnement de la menace constituent une étape clé. Cette étape représente un facteur de stress non négligeable. La pression du temps, l'irruption de nombreuses inconnues ainsi que l'arrivée en flux continu d'intervenants et de décideurs multiples contribuent à la genèse et à l'entretien d'une certaine confusion [6]. On parle de phase chaotique ou de « brouillard de guerre » [7] se traduisant par une phase de sidération initiale, inévitable, dont il faut avoir conscience. Anticiper cet état psychologique, c'est tout d'abord en accepter la composante émotionnelle afin d'être en mesure de s'y adapter de la meilleure des manières [8].

Des outils d'aide à la décision comme des procédures opérationnelles guideront les équipes à traverser cette période, sans trop obérer leurs capacités de fonctionnement [9]. Ils doivent être simples d'usage, à l'image d'une checklist [10] (Tableau 1). Il faut savoir également optimiser son potentiel d'intelligence adaptative, en tirant profit des expériences passées de décisions prises en état de stress extrême et en se préparant à affronter de nouvelles expériences $[11,12]$.

\section{Réguler ses émotions}

Pour les acteurs de l'urgence médicale préhospitalière, la participation à des opérations à haute intensité est particulièrement anxiogène. À l'incertitude évoquée précédemment vient s'ajouter pêle-mêle la nécessité de travailler en situation dégradée, avec des équipes inconnues et aux habitudes différentes, sous l'autorité initiale de forces de sécurité, laissant dans ce cas planer le doute sur une menace physique. Cette notion de « menace », au sens sécuritaire du terme, ne fait pas partie, culturellement, du vocabulaire habituel des équipes de secours et de soins préhospitaliers, même si les données sociétales contemporaines contribuent de plus en plus à les amener sur ce terrain [13]. 
Tableau 1 Check-list « $1^{\text {er }}$ départ pour tuerie de masse »

\section{- Je prépare mon intervention avec mon équipe}

J'analyse et je comprends l'environnement

Je fais un point de situation concernant d'éventuelles victimes

Je répartis les rôles de chacun dans mon équipe

Chaque membre de mon équipe est identifiable et équipé en conséquence

J'ai connaissance du canal de communication dédié à la cellule de crise Samu (tél., radio...)

Nous repérons la ZAO, le PRM

Nous connaissons l'itinéraire d'accès sécurisé des ZAO et/ou du PRM

\section{- J'arrive sur place comme premier médecin}

Je repère et je respecte le périmètre de sécurité établi par le COPG

J'identifie et je prends contact avec le premier COS

J'endosse le rôle de pré-DSM en tant que premier médecin sur place

J'établis un point de situation initial, un $1^{\text {er }}$ bilan d'ambiance à la cellule de crise en régulation médicale

Je demande des renforts médicaux et des moyens en fonction des besoins de la crise

Je participe aux points de situations interservices réguliers

\section{- J'organise la phase de sauvetage des premières victimes}

Je désigne en accord avec le COS un ou plusieurs PRV

Je désigne en accord avec le COS un ou plusieurs PMA, un PRI

J'organise avec le COS les norias d'extraction et d'évacuation

Je m'assure de la signalisation claire des différentes zones de prise en charge des victimes

J'organise l'accueil et l'orientation des flux spontanés des victimes valides après un safety check

J'organise le tri initial des victimes

Je délègue les gestes de DCR aux primo-intervenants secouristes en les adaptant au flux de victimes

J'assure la mise en place d'un listing et d'outils d'identification des victimes

Samu : Service d'aide médicale urgente ; ZAO : zone d'attente opérationnelle ; PRM : point de regroupement des moyens ; COPG : commandant des opérations de police et de gendarmerie ; COS : commandant des opérations de secours ; DSM : directeur des secours médicaux ; PRV : point de regroupement des victimes ; PMA : poste médical avancé ; PRI : point de regroupement des impliqués. DCR : damage control ressuscitation

Dans ce contexte déstabilisant car non maîtrisé, savoir optimiser son potentiel consiste en premier lieu à ne pas se laisser déborder par ses émotions, afin de réduire au maximum cette phase initiale de sidération. Celles-ci doivent être reconnues, pour soi-même et pour les autres, et leur expression acceptée [14]. Ce nécessaire travail d'anticipation peut trouver sa concrétisation dans la pratique régulière de techniques d'optimisation du potentiel [15] comme par exemple la ventilation relaxante, mais aussi par une nécessaire participation à des exercices réels et de simulations afin d'optimiser les compétences non techniques comme la confiance en soi et la communication affirmée.

\section{Définir les rôles de chacun}

Le temps de projection (de l'alerte jusqu'à l'arrivée sur place) doit être mis à profit pour briefer son équipe et permettre l'identification de chacun des membres par des chasubles, brassards ou casques nominatifs de leurs rôles respectifs. Le chef d'équipe doit commander et donner des ordres simples, précis, concis et en nombre limité (jamais plus de quatre). À titre d'exemple, le médecin se charge de la coordination avec les autres acteurs et de l'analyse de la situation. L'infirmier commence à rassurer les victimes et à réaliser des gestes de sauvetage sur les victimes accessibles, pendant que l'ambulancier s'assure du circuit d'arrivée des renforts et des évacuations. Il est fondamental de conserver une capacité d'adaptation et d'innovation, sans que cela conduise à se mettre en danger [16].

La communication est simple, précise et empathique afin d'installer un climat de confiance impératif au bon déroulement des opérations [17]. En pareilles circonstances, les capacités de mémorisation des consignes sont mises à mal. Il revient alors au médecin, chef d'équipe, de demander, à chaque membre ayant reçu un ordre, un feed-back des consignes données pour valider leur compréhension et leur intégration [18].

\section{Expliquer les règles de sécurité}

Des règles de sécurité doivent être appliquées strictement afin d'éviter la blessure d'un intervenant soignant, notamment par 
sur-attentat. Ce dernier doit être redouté et son risque connu de tous, car c'est un mode opératoire couramment employé aux Proche- et Moyen-Orient. Le médecin doit présenter à chaque membre de son équipe les principes de sécurité qui s'imposent à eux en respectant les six règles contenues dans le tableau 2. En effet, tant que la menace n'est pas totalement maitrisée, la force «menante » est la force de sécurité intérieure. C'est elle qui appréhende le mieux le risque lié à la menace et qui doit l'analyser et fixer à tous les intervenants les règles de sécurité. Les services de secours et de soins sont la force " concourante ». Le positionnement sur le terrain respecte ce principe. Le médecin responsable de l'équipe doit s'assurer que ces règles sont comprises par les membres de son équipe. Même si l'assaut est terminé, certains risques (explosif, nucléaire, radiologique, biologique, chimique [NRBC]) peuvent persister longtemps et justifier ainsi d'un périmètre de sécurité élargi et durable.

\section{Rejoindre ou définir le point de stationnement}

L'ambulancier ou le chef d'équipe se projetant sur le lieu de crise doit prendre le temps d'identifier précisément son point d'arrivée. Lorsque celui-ci a été défini (point de regroupement des moyens [PRM] ou zone d'attente opérationnelle [ZAO]), le trajet permettant de s'y rendre en sécurité est identifié et respecté. En l'absence de consigne, deux options s'offrent à l'équipe. La première consiste à rejoindre le lieu sécurisé le plus proche (commissariat, gendarmerie, centre de secours [19]). La seconde est de choisir un point de stationnement se situant sur un axe localisé à plus de deux angles droits de celui sur lequel se déroule l'événement. Le trajet emprunté doit passer le plus à distance possible du lieu du sinistre. La réflexion est la même concernant les circuits d'évacuation ou de fuite éventuelle.

\section{Anticiper les circuits d'évacuation}

L'anticipation des circuits d'évacuation, dédiée au premier médecin engagé sur le terrain, le pré-directeur des secours médicaux [pré-DSM]), lui confère une position stratégique vis-à-vis du commandant des opérations de police et de gen- darmerie (COPG) et du commandant des opérations de secours (COS) en attendant l'arrivée du directeur des secours médicaux (DSM). En restant parfaitement dans sa fonction, il s'intègre alors pleinement au dispositif. Il est fondamental de visualiser et d'organiser dans un premier temps les futurs points de regroupement des victimes (PRV), le poste médical de l'avant (PMA) ainsi que les points de regroupement des impliqués (PRI) et/ou le centre d'accueil des impliqués (CADI). En effet, les premières équipes sont rapidement confrontées à l'afflux des blessés valides et d'impliqués, et il est donc nécessaire de déterminer rapidement les circuits propres des impliqués vers les futurs PRI-CADI. Cela se fait en étroite collaboration avec le premier COS et le premier COPG, dont les priorités sont d'assurer et d'anticiper l'aspect sécuritaire et judiciaire du recueil des impliqués.

\section{Quelles doivent être mes toutes premières actions lorsque j'arrive sur place?}

\section{Mise en sécurité de l'équipe}

La situation est confuse, et la menace pas clairement identifiée et fixée. L'équipe Smur, comme les équipes de secours, peut être l'une des toutes premières équipes arrivées sur place. Celle-ci se retrouve alors dans la zone non sécurisée (menace à proximité, mouvement de foule), à initier des prises en charge de blessés. Il convient alors de ne pas exposer inutilement son équipe en réalisant un confinement de fortune. En se faisant identifier et localiser, l'équipe médicale est ensuite exfiltrée par les forces de sécurité intérieure vers la zone sécurisée. La mise en sécurité de l'équipe passe par le stationnement en zone sécurisée du véhicule, selon la procédure établie précédemment. Le médecin doit se mettre en relation avec l'officier des forces de police primointervenantes et au besoin attendre ces dernières avant de s'engager. Cet officier qui endosse le rôle du premier COPG se doit d'intégrer la problématique des secours conventionnels et proposer des options garantissant la sécurité de ces intervenants. Les informations obtenues sont immédiatement communiquées au Samu à l'intention des futurs

Tableau 2 Règles de sécurité à respecter par l'équipe soignante préhospitalière en arrivant sur la zone

- Ne pas arriver à l'adresse indiquée sans indication formelle de la Police. Éteindre dès que possible les sirènes

- Conserver son équipe groupée et protégée des dangers (murs et bâtiments)

- Rester joignable, observer et rendre compte

- Pas d'engagement ni d'initiative individuelle ou collective sans autorisation du commandant des opérations de police

et de gendarmerie (COPG)

- Communication minimale et rassurante envers les impliqués et les blessés : « vous êtes en sécurité et nous nous occupons de vous,

suivez-nous ! »

- Présence policière obligatoire pour assurer le safety check 
intervenants. Il convient donc d'être extrêmement prudent le temps de la montée en puissance des moyens et de la création d'un zonage précis.

\section{Participation au point de situation sur la menace et partage d'informations}

À l'arrivée des premières équipes de sécurité intérieure comme de secours ou de soins, le point de situation effectué par les décideurs est la priorité. Le pré-DSM se met en relation avec l'officier de police ou de gendarmerie, qui assurera le rôle de COPG, ainsi qu'avec l'officier sapeur-pompier qui assure celui de COS. Les informations partagées sont parcellaires et très évolutives, du fait du « chaos » initial. Il serait contre-productif de chercher une information exhaustive en accaparant le temps du COPG au détriment de son action de neutralisation de la menace. Les informations indispensables à partager dès l'arrivée sur place concernent l'état de la situation et figurent dans le tableau 3. À ce stade, les questions d'ordre médical (typologie des blessures, gravité des blessés, identification) sont prématurées. La notion de blessés et une estimation de leur nombre sont suffisantes.

Les informations ainsi collectées sont transmises à la régulation médicale du Samu pour une montée en puissance

\begin{tabular}{|ll|}
\hline Tableau 3 Synthèse de l'analyse de la situation \\
\hline \multicolumn{1}{|c|}{ État de la situation } \\
\hline Types de menace ? & Balistique \\
& Engins explosifs \\
& Engins incendiaires \\
& Agents NRBC \\
& Autres \\
Typologie de la menace ? & Tuerie de masse en cours \\
& Tireur isolé \\
& Attaque suicide \\
& Périple meurtrier \\
Évolutivité ? & Prise d'otages \\
& Non évaluable \\
& Maitrisée \\
Cinétique ? & Fixée \\
& Terminée \\
& Rapide \\
Existe-t-il & Lente \\
une cartographie & Indéterminée \\
des victimes ? & Points de regroupement \\
& spontané de blessés \\
& Axes de fuite victimes cachées \\
& Circuits d'évacuations \\
& spontanées \\
NRBC : nucléaire radiologique biologique chimique \\
\end{tabular}

selon les plans de secours dédiés. La régulation médicale du Samu s'assure que l'ensemble des équipes Smur en cours d'acheminement vers le théâtre des opérations dispose des dernières informations relatives à la sécurité, idéalement sous forme d'un bilan d'ambiance radio partagé par tous sur le « canal santé ». Ces informations sont également partagées par le pré-DSM avec toutes les équipes médicales qui sont amenées à intervenir et respectent les consignes fixées par le COPG. Le médecin sur place fera remonter à la régulation médicale du Samu les actions menées.

Le leadership du premier médecin sur place doit permettre de prendre les décisions indispensables à l'engagement tactique, en sécurité, des équipes arrivées en renfort. Ce premier médecin agit comme pré-DSM et définit la stratégie médicale, en particulier la mise en œuvre de mesures de damage control préhospitalier si la situation l'impose. À l'inverse, il s'abstient d'initier pareilles mesures si la situation ne l'exige pas d'un point de vue médical. En cas de blessure par arme de guerre, il applique la conduite à tenir pour les professionnels de santé émanant de la direction générale de la Santé [20].

\section{Accès aux victimes}

La perte de chance pour les blessés en choc hémorragique induite par le temps qui s'écoule conduit les soignants à souhaiter la réalisation au plus vite de gestes de sauvetage, en particulier d'hémostase, et pour ce faire d'une évacuation vers un bloc opératoire. Aucune opération de " sauvetage " sous la menace ne peut être réalisée sans l'autorisation du COPG et, le cas échéant, elle doit s'organiser en étroite collaboration avec le COS qui dirige les manœuvres. En dehors du risque qu'une telle action fait courir au soignant, l'ensemble du dispositif de sécurité, de secours et de soins peut être mis en danger, par exemple si cet acte de soin conduit au déclenchement d'un engin explosif sur une victime piégée, voire à la constitution d'une prise d'otages.

Il existe différents moyens de porter secours sans s'exposer, dont la pertinence est évaluée à mesure de l'évolution de la crise, par exemple en conseillant les forces de sécurité intérieure dans les décisions de manœuvres d'extraction d'urgence. Des paroles rassurantes guidant la réalisation de gestes de sauvetage à distance en zone d'exclusion peuvent dans des cas extrêmes être prononcées par les équipes de secours. Ces gestes salvateurs peuvent être réalisés en zone d'exclusion soit par les victimes valides elles-mêmes, sur elles ou sur les victimes avoisinantes, soit par les forces de police primo-intervenantes directement en contact avec les blessés. Par ailleurs, un appel ferme et directif à la voix permet de regrouper les victimes et de les faire venir vers soi $[21,22]$. En plus de faciliter le rapprochement des victimes du lieu de prise en charge médicale, cette façon de procéder permet un premier tri particulièrement efficace en identifiant 
les personnes valides. Elle doit impérativement se faire en présence de forces de sécurité qui assurent une fouille de sécurité, ou safety check, avant toute prise en charge soignante. En effet, un terroriste peut se glisser volontairement parmi les victimes dans le but d'atteindre le dispositif de secours et de soins pour générer un sur-attentat.

Enfin, il faut envisager de demander aux forces de sécurité intérieure s'il leur est possible de procéder à l'extraction des victimes les plus proches et/ou les moins exposées. La situation est initialement trop instable pour constituer un ou des corridors d'extraction bien définis. Une analyse pragmatique de la situation est impérative à chaque instant de la crise.

\section{Comment réagir lors de l'arrivée des forces d'intervention spécialisée ?}

C'est en principe lors de l'arrivée des forces d'intervention spécialisée (RAID, GIGN, BRI PP) ou des forces de sécurité intérieure de niveau 2 (Brigade de recherche et d'intervention [BRI], Compagnie de sécurité intérieure [CSI], Compagnie républicaine de sécurité [CRS], Peloton de surveillance et d'intervention de la Gendarmerie [PSIG]) que la situation va commencer à se clarifier et permettre de s'organiser. Cette étape nécessite un nouveau point de situation permettant de préciser et de répondre aux questions figurant dans le tableau 4.

\section{Création de points d'extraction des victimes}

L'arrivée des forces d'intervention spécialisée ne modifie pas immédiatement le zonage mis en place. Néanmoins, leur action va permettre de repousser la menace et de mettre en sécurité les victimes. Rappelons qu'il s'agit d'une action prioritaire sur toutes les autres, y compris le secours comme le soin. En repoussant la menace, les forces d'intervention spécialisée font bouger les « frontières » et convertissent une partie de la zone d'exclusion en zone contrôlée. Le ou les points d'extraction des victimes (PEV) matérialiseront les points les plus avancés, marquant les limites à ne jamais dépasser par les personnes non habilitées, sous peine d'exposition au danger. Une signalétique, par dispositif lumineux clignotant ou bannière drapeau, est utilisée par les forces d'intervention spécialisée (Fig. 1) afin d'identifier les PEV.

La zone d'exclusion n'est accessible qu'aux personnes habilitées à évoluer en milieu hostile, telles que les forces d'intervention spécialisée et les forces de sécurité intérieure de niveau 2. Ces équipes procéderont rapidement au sauvetage et à la mise en sécurité des victimes au niveau d'un premier point, propre aux forces d'intervention spécialisée, où se trouvent les médecins tactiques de " reprise ». Ces derniers réalisent un prétriage qui a pour objectif principal la mise en sécurité des victimes. Cet exemple d'algorithme de secours tactique s'inspire du simple triage and rapid treatment (START) (Fig. 2). Il aide la prise de décisions sur la base de critères tactiques et de sauvetage ( $«$ le bon

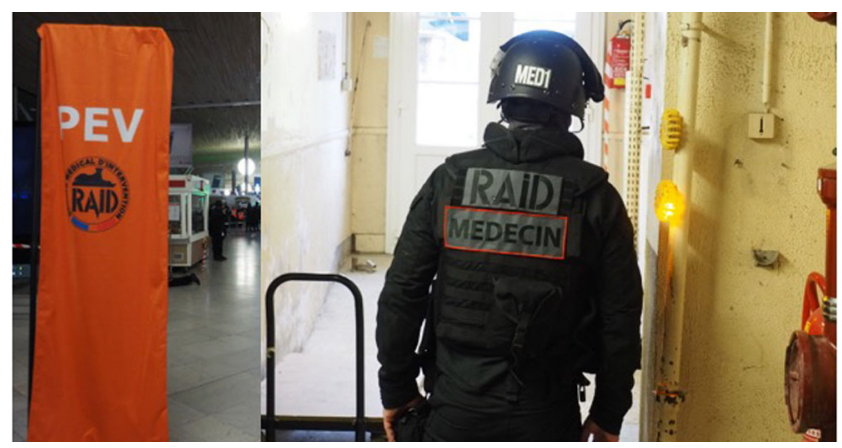

Fig. 1 Signalétiques (fanion orange et gyrophare orange) d'un point d'extraction des victimes (PEV)

Tableau 4 Synthèse point de situation initiale-cartographie secours

Point de situation initiale-Forces d'intervention spécialisée-Secours

Le zonage est-il déterminé et compris ?

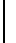

Est-ce que les sites de prise en charge des victimes sont identifiés?

Est-ce que les équipes d'extraction sont constituées et identifiées?

Estimation des modalités d'extraction
Zone d'exclusion police (ZEP)

Zone contrôlée (ZC)

Zone soutien (ZS)

Point d'extraction des victimes (PEV)

Point de regroupement des victimes (PRV)

Poste médical avancé (PMA)

Point de regroupement des impliqués (PRI) et centre d'accueil

des impliqués (CADI)

Un chef par équipe identifié

Interlocuteur identifié

Un PEV d'attribution et de positionnement

Estimation des flux

Estimation des délais 
Algorithme décisionnel de secourisme tactique

en cas d'afflux massif de victimes avec persistance de la menace
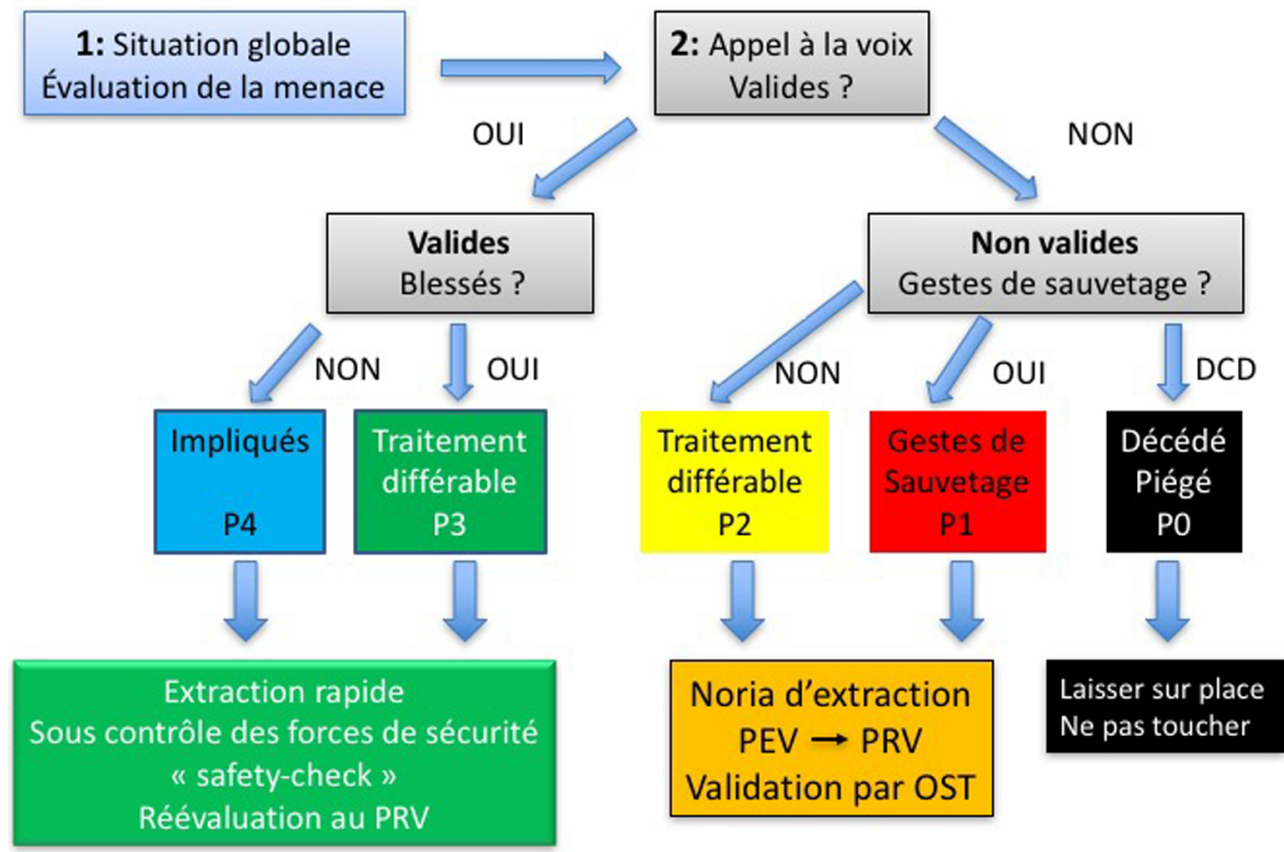

Fig. 2 Exemple de prétriage tactique en cas d'afflux de victimes sous la menace : P0 : priorité 0 ; P1 : priorité 1 ; P2 : priorité 2 ; P3 : priorité 3 ; P4 : priorité 4 ; PRV : point de regroupement des victimes ; PEV : point d'extraction des victimes ; OST : officier de secours tactique

geste au bon endroit au bon moment ») et non uniquement de critères médicaux. Pour fluidifier les circuits et la communication, des codes couleurs d'identification de tri tactique attribués à chaque victime par le GIGN et le RAID (Fig. 3) correspondent aux codes couleurs OTAN $[23,24]$ et à ceux utilisés par la direction générale de la Sécurité civile et de la gestion de crise.

Dans cette zone de reprise, l'extraction des victimes vers les PEV est réalisée par les forces de sécurité intérieure après un safety check et sous le contrôle des médecins tactiques qui font office d'accélérateurs de flux. Ils sont aussi les « yeux » des secours et équipes médicales conventionnels pour anticiper la communication des informations au COS et son DSM.

\section{Création de corridors d'extraction et organisation de la zone contrôlée}

La zone contrôlée est évolutive. Elle s'adapte à la menace et s'élargit au fur et à mesure que les forces d'intervention spécialisée repoussent la menace. On y trouve uniquement les équipes secouristes d'extraction. Les équipes médicales restent positionnées au PRV à la limite de cette zone contrôlée. Le PEV constitue donc le point le plus avancé de la zone contrôlée et le PRV, son point le plus reculé, donc sécurisé.

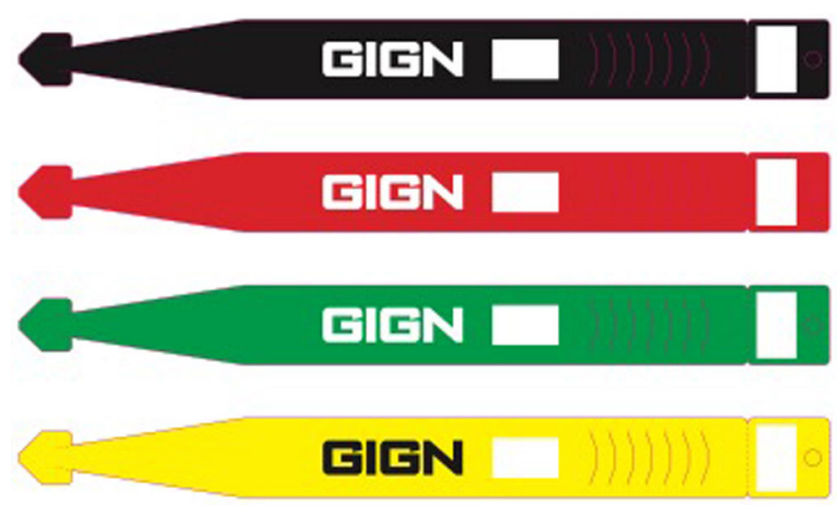

Fig. 3 Bracelets de triage tactique utilisés par le Groupe d'intervention de la Gendarmerie nationale [GIGN]

Ce cheminement dans la zone contrôlée entre PEV et PRV forme un corridor d'extraction. Les équipes d'extraction évoluant dans ce "sas » sont numérotées en fonction du PEV d'attribution : PEV 1, 2, 3, etc. La zone contrôlée est sous la responsabilité du COS, mais son accès et sa sécurité sont sous la responsabilité du COPG. L'organisation des circuits d'extraction et les manœuvres dynamiques de déplacement des PEV sont validées au poste de commandement (PC) tactique entre l'officier de liaison sapeur-pompier (OLSP) et l'« officier de secours tactique » (OST) des forces 
d'intervention spécialisée. Sur ordre de ce dernier, l'OLSP fait progresser les équipes d'extraction de manière très dynamique. Pour remplir cette mission, ces équipes doivent donc être particulièrement mobiles : plusieurs (trois ou quatre maximum) binômes avec un lot de damage control et un brancard d'extraction adapté.

Ainsi, les forces de sécurité et les équipes secouristes d'extraction évoluant en zone contrôlée suivent les consignes de l'OST via l'OLSP et le COS qui donnent le tempo des extractions et de l'avancée progressive des PEV, grâce aux retours d'informations des médecins tactiques évoluant en zone d'exclusion police. La synchronisation des actions de chacun permet un gain de temps conséquent sur l'extraction des victimes en zone sécurisée où elles sont rapidement prises en compte par les équipes médicales préhospitalières dès le passage par les PRV. Un canal de communication radio « santé» partagé est à privilégier pour simplifier ces manœuvres. Cette fluidité accélère l'évacuation des blessés vers les structures hospitalières définies par la régulation médicale du Samu pour qu'elles bénéficient de gestes d'hémostase définitifs.

\section{Anticipation, réactivité et adaptation}

La réponse « secours-soins » doit donc être dynamique et adaptative. Les équipes médicales préhospitalières doivent se tenir prêtes à prendre en charge des flux de victimes n'ayant bénéficié au mieux que de gestes de " sauvetage " et d'un prétriage. Pour adapter leur réponse, elles doivent préalablement anticiper et visualiser mentalement la mission à venir, grâce aux informations que leur a communiquées le pré-DSM (ou le DSM de site une fois celui-ci installé dans ses fonctions). Cette préparation mentale se focalise uniquement sur l'opération de triage et la réalisation des gestes médicaux de sauvetage strictement indispensables. Elle permet de ne pas se faire parasiter par l'environnement le moment venu et de contrôler les flux. Elle contribue à économiser ses forces lorsque l'engagement, nécessitant célérité, intensité physique et psychologique, sera total.

Les équipes de secours évoluant entre le PEV et le PRV doivent être en mesure de se déplacer rapidement grâce à un entraînement régulier et être dotées d'un matériel adapté. Pour des raisons de sécurité comme d'efficience, la fluidité et l'adaptabilité des manœuvres doivent être sans compromis. Pour cela, il est important de se concentrer uniquement sur sa zone de « travail ». Seules les informations pertinentes au bon déroulement de l'action de chacun sont transmises par les forces d'intervention spécialisée aux leaders de chaque maillon de la chaîne de secours et de soins.

\section{Prérégulation médicale}

Raccourcir les délais de prise en charge à l'hôpital passe non seulement par l'identification des circuits d'évacua- tion, mais également par le choix et la prérégulation médicale des établissements de soins receveurs par les régulateurs du Samu. Cette prérégulation médicale se fonde sur les flux capacitaires préalablement déclarés par les établissements de santé. Ces derniers ayant validé en planification leurs flux capacitaires respectifs, l'action urgente pour le Samu est de les alerter pour les informer de la menace et leur demander de se mettre en capacité de recevoir les victimes. Pour cette phase, le Samu concentre donc son temps uniquement sur l'information des hôpitaux et non plus sur les recherches de places. L'équipe sur le terrain bénéficie du recueil capacitaire préétabli, et le médecin régulateur du Samu sur place décide de la destination des patients vers l'établissement le mieux adapté à la pathologie du patient et d'après les données capacitaires préétablies, sans attendre le retour d'information de la régulation médicale. Cela lui permet d'anticiper puis d'accélérer le parcours du patient, que l'on sait « attendu ». Cette approche a fait la preuve de sa pertinence [3]. Les circuits ainsi prérégulés permettent de diminuer les "morts évitables » consécutives à un retard de prise en charge $[25,26]$. Cette prérégulation médicale exerce par ailleurs un effet rassurant pour les intervenants sur le terrain. Le médecin régulateur rend compte dès que possible à la régulation médicale du Samu de l'utilisation des places. La traçabilité des patients et leur identification sont, quant à elles, assurées par la mise en place de bracelets du système d'information numérique standardisé (SINUS) avant l'évacuation vers les hôpitaux. Il convient de communiquer à la régulation médicale et au COPG l'éventuelle saturation d'axes de circulation traversés pendant la phase de projection ou d'évacuation. À l'arrivée à l'hôpital, la saisie des informations administratives dans le logiciel Système d'information victimes (SIVIC) doit permettre l'identification, la traçabilité et l'actualisation des capacités de prise en charge des établissements dans les outils de gestion de crise des Samu.

\section{Comment, dans ce contexte, je soigne les premières victimes ?}

Dans ce dernier chapitre figurent les trois points que doit connaître tout médecin positionné au PRV.

\section{Analyse du flux et prétriage}

Le médecin situé au PRV doit, dans un premier temps, analyser l'arrivée des blessés du ou des PEV. La compréhension des (af)flux de victimes arrivant (cinétique et intensité) prime sur les soins. Cette phase de discernement permet l'adaptation des moyens en aval (du PEV à l'hôpital, en passant par les PRV, PMA et moyens Smur). Elle est partagée avec le COS. 
L'inadéquation entre les moyens capacitaires et le nombre de victimes impose une adaptation de l'organisation.

Le triage tactique réalisé dans la zone d'exclusion police par le soutien médical tactique des forces d'intervention spécialisée, matérialisé par les bracelets (Fig. 3), constitue un outil précieux d'aide à la décision et à l'organisation. Ces bracelets facilitent la transmission d'informations entre les médecins tactiques en zone d'exclusion et les services de secours en zone de soutien.

\section{Quels gestes thérapeutiques au PRV ?}

Dans le cas d'une tuerie de masse avec une menace non mâ̂trisée, les actions de secours et de soins des primointervenants dans la zone d'exclusion ainsi que durant la phase de soutien dans la zone contrôlée n'autorisent que des techniques de sauvetage $[26,27]$. Chaque geste doit tenir compte de l'équilibre entre le bénéfice pour le patient, d'une part, et la menace et la cinétique du flux potentiel à venir de victimes, d'autre part. L'efficacité de la prise en charge globale des victimes consiste en la réalisation de gestes efficaces et rapides permettant une mobilisation et une extraction rapide des blessés. La visualisation mentale et l'anticipation préalable de la situation permettent de préparer les équipes à mâ̂triser les rares gestes techniques pertinents devant être effectués dans ces situations, telles la pose de garrots tactiques, de pansements compressifs, de pansements thoraciques, l'exsufflation de pneumothorax, la mise en place de clamps vasculaires, la libération des voies aériennes supérieures [28].

La typologie de la mission conditionne le matériel emporté comprenant, outre les lots de damage control, des brancards simples d'extraction et la signalétique pour un prétriage des victimes. Tout le matériel non indispensable devra être laissé dans la ZAO ou dans les véhicules d'intervention.

\section{Accélérer les flux d'évacuation vers la zone de soutien}

Aux soins de sauvetage à réaliser vient s'ajouter l'orientation des victimes, avec comme impératif que cette orientation soit rapide vers un lieu sécurisé adapté à leur état. Il faudra hâter l'acheminement au PRV des patients les plus graves, afin qu'ils y bénéficient d'une prise en charge salvatrice et fassent l'objet d'une orientation vers le centre hospitalier adapté à leur état.

\section{Conclusion}

Lors d'une intervention préhospitalière pour tuerie de masse, les équipes Smur sont sous tension dès leur déclenchement. Les soins ne constituent qu'une partie de leur mission, et en général, c'est l'organisation qui occupe le premier plan. Les équipes de secours et de soins primo-intervenantes, en accord avec le COS, agissent comme de véritables accéléra- teurs de flux d'évacuation des blessés. Pour ce faire, elles identifient les problèmes (menace, nombre de victimes et accès à ces dernières) qu'elles partagent avec la régulation médicale du Samu, dans le but d'adapter en sécurité la montée en puissance tout comme le circuit d'aval des blessés. Elles assurent le tri et les soins aux victimes, tel qu'elles l'ont appris. L'ensemble de ces actions se fait dans un contexte dégradé, forcément anxiogène. Pour surmonter ces difficultés, elles font appel à des techniques de gestion du stress, à des entraînements et à de la simulation.

Liens d'intérêts : Mathieu Raux organise une formation de développement professionnel continu au sein de Sorbonne université en collaboration avec le Service médical du RAID.

\section{Références}

1. Turner CDA, Lockey DJ, Rehn M (2016) Pre-hospital management of mass casualty civilian shootings: a systematic literature review. Crit Care 20:1-11

2. Butler FK, Bennett B, Wedmore CI (2017) Tactical combat casualty care and wilderness medicine: advancing trauma care in austere environments. Emerg Med Clin North Am 35:391-407

3. Service médical du RAID (2015) Tactical emergency care during hostages' crisis: care principles and feedback. Ann Fr Med Urgence 5:166-75

4. Federal Emergency Management Agency (2017) Active shooter and mass casualty incidents. September 2013. USFA FEMA. https://Usfa.fema.gov/downloads/pdf/publications/active shooter_guide.pdf (Dernier accès $1^{\mathrm{er}}$ juin 2018)

5. Société française de médecine d'urgence, Société française d'anesthésie et de réanimation, Société française de médecine de catastrophe, Société française de chirurgie d'urgence (2018) Recommandations formalisées d'experts : prise en charge d'une tuerie de masse. Ann Fr Med Urgence 8:[in press]

6. Young DL, Goodie AS, Hall DB, et al (2012) Decision making under time pressure, modeled in a prospect theory framework. Organ Behav Hum Decis Process 118:179-88

7. Dubourg O, Retail Du C, Fressancourt Y, et al (2017) Principes tactiques et de médicalisation sur les lieux d'un attentat. In: Mérat S, Pasquier P (eds) Le blessé par attentat terroriste. Arnette, Montrouge, pp 67-78

8. La Porte TR (2015) Organized social complexity: challenge to politics and policy. Princeton University Press, Princeton

9. Rimstad R, Sollid SJ (2015) A retrospective observational study of medical incident command and decision-making in the 2011 Oslo bombing. Int J Emerg Med 8:4

10. Hicks C, Petrosoniak A (2018) The human factor: optimizing trauma team performance in dynamic clinical environments. Emerg Med Clin North Am 36:1-17

11. Ministère de la Défense. Circulaire 4725/ARM/EMA/CNSD/EIS/ DGF du 14 décembre 2017 relative à la formation et à l'enseignement des techniques d'optimisation du potentiel au sein des forces armées. https://www.bo.sga.defense.gouv.fr/boreale_internet/ download.php?f type $=$ PDF\&f id $=$ MjY1MTE0LnBkZg $==\&$ no cache $=2$ (Dernier accès le $1^{\text {er }}$ août 2018)

12. Ozel F (2001) Time pressure and stress as a factor during emergency egress. Saf Sci 38:95-107

13. Berkovits A, Tillant D, Hoang P (2004) La violence aux urgences. Gestions Hospitalières 434:39-43 
14. Perreaut-Pierre E (2012) Comprendre et pratiquer les techniques d'optimisation de potentiel. InterEditions, Paris

15. Lauria MJ, Gallo IA, Rush S, et al (2017) Psychological skills to improve emergency care providers' performance under stress. Ann Emerg Med 70:884-90

16. Maule AJ, Hockey GR, Bdzola L (2000) Effects of time-pressure on decision-making under uncertainty: changes in affective state and information processing strategy. Acta Psychol 104:283-301

17. Combalbert L (2018) Le management des situations de crise. Anticiper les risques et gérer les crises. ESF éditeur, Paris

18. Paquette G (1987) Feedback, rétroaction, rétroinformation, réponse... du pareil au même. Communication et Langages 73:5-18

19. Ministère de l'Intérieur. Arrêté préfectoral du 2 janvier 2017. Plan départemental Tuerie de masse. Acte de terrorisme annexé au dispositif ORSEC départemental au titre des dispositions spécifiques http://www.savoie.gouv.fr/content/download/19621/160184/file/ 2017.01.10\%20-\%20RAA\%20N\%C2\%B073-2017-005.pdf (dernier accès le 06/09/2018)

20. Direction générale de la Santé (2018) Agressions collectives par armes de guerre : conduites à tenir pour les professionnels de santé. http://solidarites-sante.gouv.fr/IMG/pdf/Agressions-collectives.pdf (Dernier accès le 19 juillet 2018)

21. Langlois M, Ploquin F (2016) Médecin du RAID : vivre en état d'urgence. Albin Michel, Paris
22. Service médical du RAID (2016) Retour d'expérience des attentats du 13 novembre 2015. Gestion de l'attaque terroriste du Bataclan par les médecins d'intervention de la Police nationale. Ann Fr Med Urgence 6:3-8

23. Lampi M, Vikström T, Jonson CO (2013) Triage performance of Swedish physicians using the ATLS algorithm in a simulated mass casualty incident: a prospective cross-sectional survey. Scand J Trauma Resusc Emerg Med 21:90

24. van Rein EAJ, Houwert RM, Gunning AC, et al (2017) Accuracy of prehospital triage protocols in selecting severely injured patients: a systematic review. J Trauma Acute Care Surg 83:328-39

25. Callaway DW, Smith ER, Cain J, et al (2011) Tactical emergency casualty care (TECC): guidelines for the provision of prehospital trauma care in high threat environments. J Spec Oper Med 11:1-20

26. Kotwal RS, Montgomery HR, Miles EA, et al (2017) Leadership and a casualty response system for eliminating preventable death. J Trauma Acute Care Surg 82:S9-S15

27. Tourtier JP, Palmier B, Tazarourte K, et al (2013) The concept of damage control: extending the paradigm in the prehospital setting. Ann Fr Anesth Reanim 32:520-6

28. Jacobs LM (2014) Joint committee to create a national policy to enhance survivability from mass casualty shooting events: Hartford Consensus II. J Am Coll Surg 218:476-8 